To appear in the Astronomical Journal, March 2003 issue

\title{
Variability-selected quasars behind the Small Magellanic Cloud ${ }^{1}$
}

\author{
A. Dobrzycki ${ }^{2}$, L. M. Macri ${ }^{3,4}$, K. Z. Stanek ${ }^{2}$, and P. J. Groot ${ }^{5}$
}

\begin{abstract}
We present followup spectroscopic observations of quasar candidates in the Small Magellanic Cloud selected by Eyer from the OGLE database. Of twelve observed objects identified as "QSO Candidate", five are confirmed quasars, with the emission redshifts ranging from 0.28 to 2.16. Two of those quasars were also recently identified independently in the MACHO database by Geha et al. We discuss the prospects of using variability-based selection technique for quasar searches behind other dense stellar fields. An additional criterion utilizing the color-color diagram should reduce the number of stars in the candidate lists.
\end{abstract}

Subject headings: Magellanic Clouds — quasars: individual

\section{Introduction}

Searches for quasars in dense stellar fields — such as the Magellanic Clouds — were in the past hampered by difficulties in selecting candidates. Optical followup on X-ray selected objects had to deal with large number of candidates in X-ray source error boxes, while variability studies required monitoring of vast number of objects. At the same time, such quasars are of great astrophysical interest, for example as reference points for analysis of

\footnotetext{
${ }^{1}$ Based on observations collected at the Magellan Baade 6.5-m telescope.

${ }^{2}$ Harvard-Smithsonian Center for Astrophysics, 60 Garden Street, Cambridge MA 02138, USA, [adobrzycki,kstanek]@cfa.harvard.edu.

${ }^{3}$ Kitt Peak National Observatory, National Optical Astronomy Observatory, 950 North Cherry Avenue, P.O. Box 26732, Tucson, AZ 85726-6732, USA, lmacri@noao.edu.

${ }^{4}$ Hubble Fellow

${ }^{5}$ Department of Astrophysics, University of Nijmegen, PO Box 9010, 6500 GL Nijmegen, The Netherlands, pgroot@astro.kun.nl.
} 
proper motions and as background sources for absorption studies. Until recently, only a handful of confirmed quasars behind the LMC and SMC were known (Blanco \& Heathcote 1986; Crampton et al. 1997; Tinney et al. 1997; Anguita, Loyola, \& Pedreros 2000; see also Kahabka et al. 1999, Haberl et al. 2000 and Kahabka, de Boer, \& Brüns 2001). Almost all of those quasars were located behind the outer, sparse parts of the Clouds.

Recent developments - such as the launch of the Chandra X-ray Observatory with its superb spatial resolution in X-rays, and availability of large photometry databases (OGLE, MACHO) - are now making systematic searches for quasars in dense stellar fields possible. Recently, Dobrzycki et al. (2002) found four X-ray quasars among serendipitous sources in four Chandra observations of objects in the LMC coinciding with the OGLE fields.

A characteristic that was often used in quasar surveys was their irregular variability. Several such surveys were performed or are on-going (Hawkins 1983; Meusinger \& Brunzendorf 2001; Rengstorf et al. 2001). In the Magellanic Clouds, this technique has been used by Geha et al. (2002), who published a list of forty seven quasars behind the LMC and SMC, selected from the MACHO database.

Between 1997 and 2001, large parts of the Magellanic Clouds were monitored for microlensing events by the Optical Gravitational Lensing Experiment (OGLE-II: Udalski, Kubiak \& Szymański 1997). Udalski et al. (1998) released BVI photometry and astrometry of 2.2 million objects from the central parts of the $\mathrm{SMC}^{6}$. In addition, a large catalog of 68,000 variable objects observed by OGLE-II in both the LMC and the SMC was prepared by Żebrun et al. $(2001)^{7}$, based on a version of the image subtraction software (Alard \& Lupton 1998) developed by Woźniak (2000).

Eyer (2002) presented an algorithm for selecting quasar candidates from objects in the OGLE database. He searched the database for slowly and irregularly varying blue objects and identified QSO candidates ("QCs") towards the Magellanic Clouds: 118 QCs towards the LMC and 15 towards the SMC. Eyer also identified several "Unclassified" objects, which had similar light curve characteristics.

In 2002 September, we performed followup observations of twelve brightest "QSO Candidates" from the SMC from the Eyer's list with the Magellan 6.5-meter Baade telescope. Five of them turned out to be previously unknown quasars; an excellent success rate. We also observed all four of Eyer's "Unclassified" objects in the direction of the SMC and none of them turned out to be a QSO.

\footnotetext{
${ }^{6}$ Data available from ftp://bulge.princeton.edu/ogle/ogle2/maps/smc/

${ }^{7}$ Data available from http://bulge.princeton.edu/ ogle/ogle2/dia/
} 
Coincidentally, less then a week after our observations had been completed, the paper by Geha et al. (2002) was posted on astro-ph. In this paper, the authors performed an analysis of the MACHO variable star database. They selected 360 quasar candidates behind the Magellanic Clouds, and completed followup observations of 259 of them. In that way, they identified forty seven quasars: thirty eight behind the LMC and nine behind the SMC. Three of their SMC objects were on the list of Eyer's candidates: two quasars and a Be star.

In this paper, we present the identifications of the new quasars behind the SMC, and we discuss the prospects for application of Eyer's method for quasar searches behind other dense stellar fields.

\section{Observations and identifications}

The optical spectra were obtained on 2002 September 16-18 with the Magellan Baade 6-5 meter telescope. We used the LDSS-2 imaging spectrograph, with a $2048 \times 2048$ SITe\# 1 CCD with a scale of $0.38 \mathrm{arcsec} / \mathrm{pixel}$, a gain of $1 e^{-} / \mathrm{ADU}$, and a readout noise of $7 e^{-}$. The slit width was 1.03 arcsec and the grism setting was $300 \mathrm{l} / \mathrm{mm}$, yielding a nominal resolution of $13.3 \AA$ A. Exposure times ranged from 120 to 600 seconds. All observations were carried out with the slit oriented in the east-west direction. Additionally, we observed two spectrophotometric standards, LTT 1788 and LTT 7379 (Hamuy et al. 1992). Following each observation, a He-Ne arc lamp spectrum was acquired for wavelength calibration purposes. Spectra were reduced in the standard way using IRAF.

Figure 1 shows the spectra of twelve of Eyer's QC objects. There are five confirmed quasars among them, and we show their spectra on the left panel, while on the right panel one can find spectra of objects that turned out to be stars. We summarize the object properties

in Table 1. We also observed all four of Eyer's "Unclassified" objects, which all turned out to be stars; for completeness, we include this information in Table 1.

The spectra of all five quasars show at least two emission lines, enabling unambiguous determination of emission redshifts. As mentioned earlier, two of those quasars (QSO J010234.69-725424.1 and QSO J010721.61-724845.5) were independently identified by Geha et al. (2002).

\section{Discussion}

We have identified five variability-selected quasars among twelve brightest Quasar Candidates identified by Eyer (2002) based on the characteristics of their light curves in the 

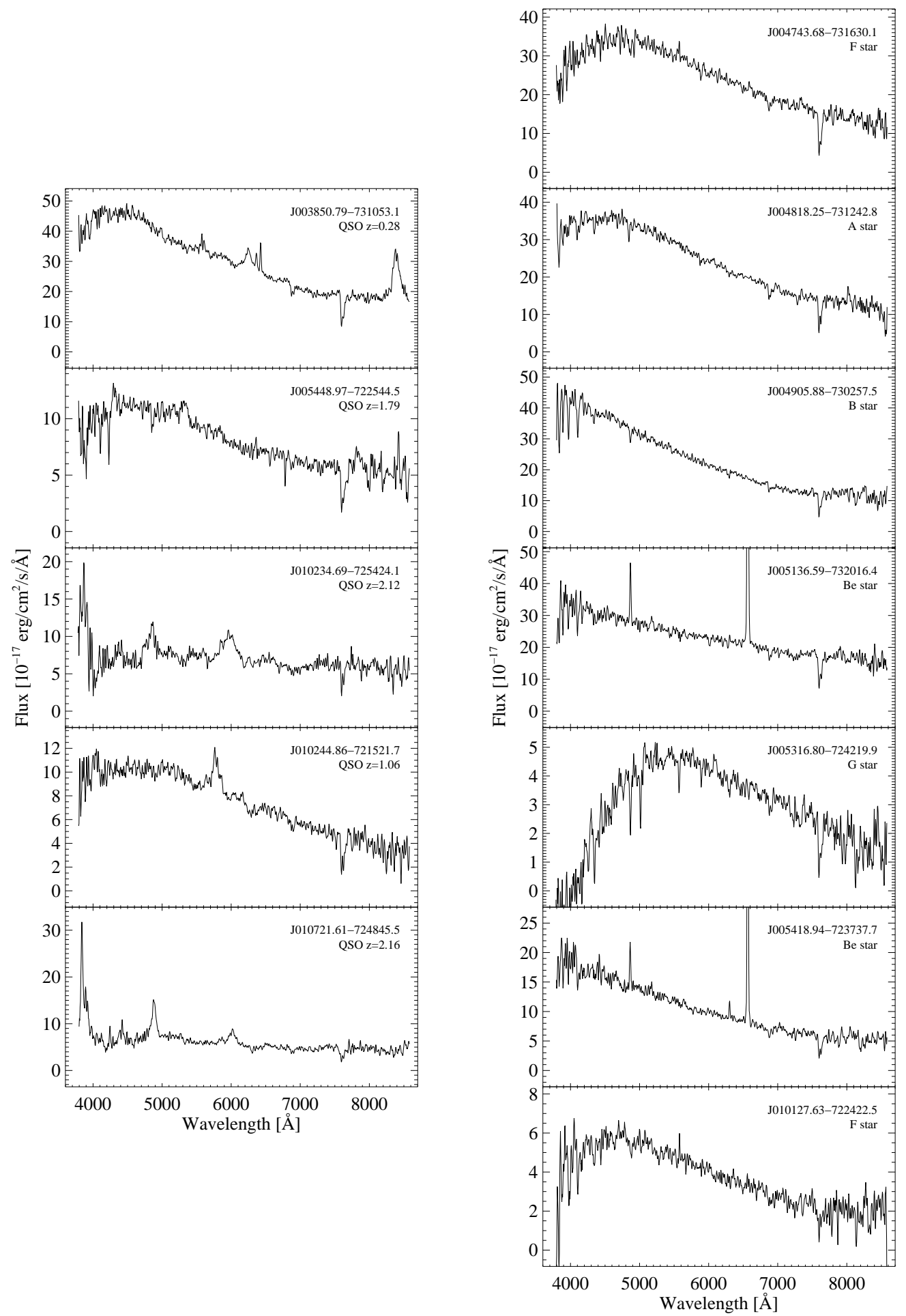

Fig. 1. - Magellan spectra of twelve brightest "Quasar Candidates" from Eyer (2002). Left panel shows identified quasars, right panel shows objects that turned out to be stars. 

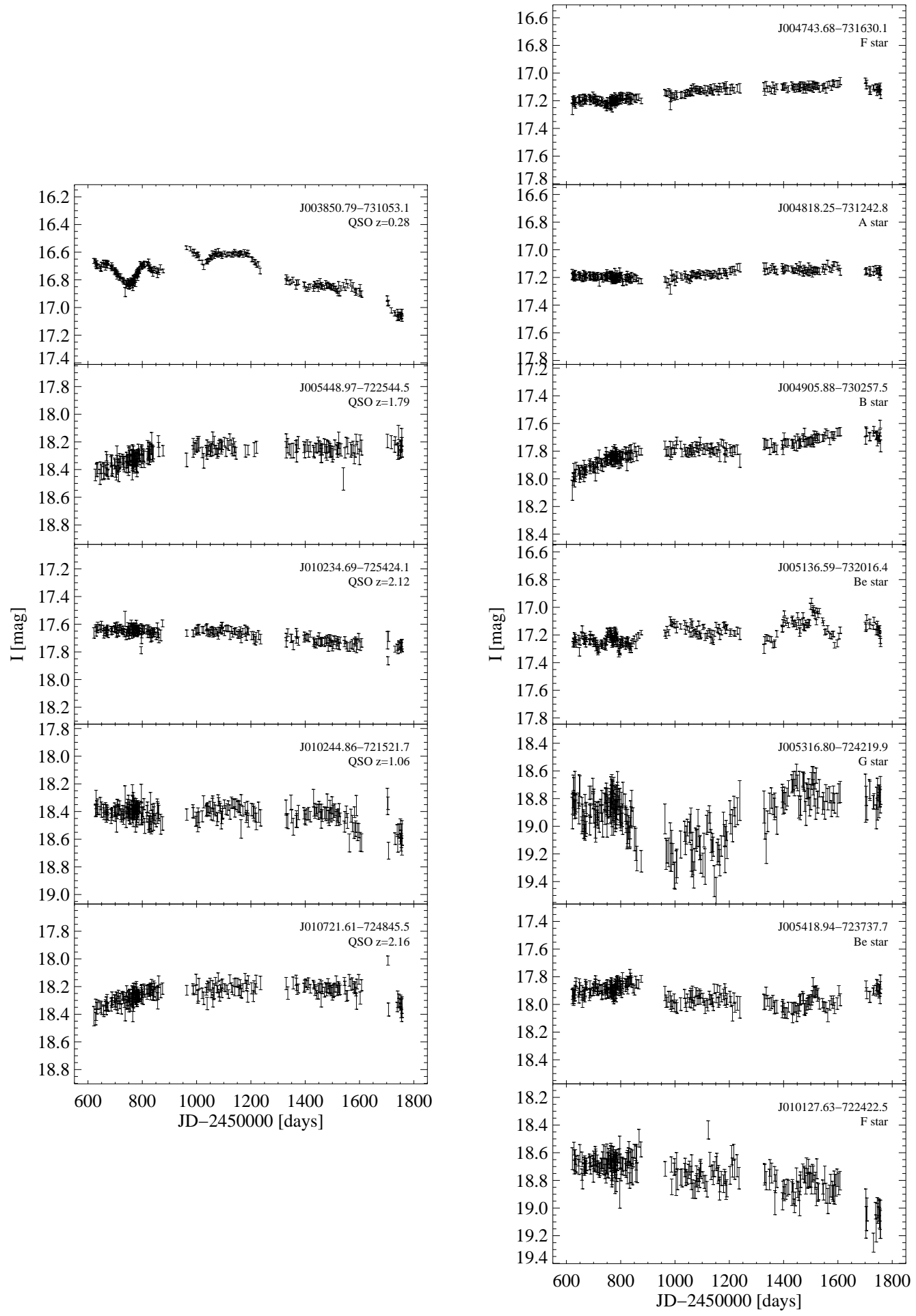

Fig. 2.- OGLE (Żebruń et al. 2001) light curves for objects from Figure 1, arranged in a similar way. JD 2,450,000 corresponds to UT 1995 October 9. 
Table 1. Object identifications.

\begin{tabular}{|c|c|c|c|c|c|}
\hline OGLE ID ${ }^{\mathrm{a}}$ & $\begin{array}{c}V^{\mathrm{b}} \\
{[\mathrm{mag}]}\end{array}$ & $\begin{array}{c}B^{\mathrm{b}} \\
{[\mathrm{mag}]}\end{array}$ & $\begin{array}{c}I^{\mathrm{b}} \\
{[\mathrm{mag}]}\end{array}$ & Eyer ID & Identification \\
\hline \multicolumn{6}{|c|}{ Quasar Candidates } \\
\hline 003850.79-731053.1 & 17.683 & 17.870 & 16.822 & S1 & $\mathrm{QSO}, z_{\mathrm{em}}=0.28$ \\
\hline 004743.68-731630.1 & 17.532 & 17.666 & 17.211 & S3 & F star \\
\hline 004818.25-731242.8 & 17.446 & 17.493 & 17.193 & $\mathrm{~S} 4$ & A star \\
\hline $004905.87-730257.5$ & 17.973 & 18.002 & 17.833 & S5 & B star \\
\hline 005136.59-732016.5 & 18.036 & 18.349 & 17.241 & S6 & Be star \\
\hline 005316.80-724219.9 & 19.263 & 19.432 & 18.860 & S7 & G star \\
\hline $005418.96-723737.7^{\mathrm{c}}$ & 18.137 & 18.194 & 17.893 & S8 & Be star \\
\hline $005448.97-722544.6$ & 19.001 & 19.180 & 18.263 & S9 & $\mathrm{QSO}, z_{\mathrm{em}}=1.79$ \\
\hline $010127.64-722422.6$ & 19.058 & 19.191 & 18.673 & $\mathrm{~S} 13$ & F star \\
\hline $010234.69-725424.1^{\mathrm{c}, d}$ & 18.346 & 18.689 & 17.640 & $\mathrm{~S} 12$ & $\mathrm{QSO}, z_{\mathrm{em}}=2.12$ \\
\hline $010244.89-721521.7$ & 18.892 & 19.385 & 18.412 & S11 & $\mathrm{QSO}, z_{\mathrm{em}}=1.06$ \\
\hline $010721.61-724845.5^{\mathrm{c}, e}$ & 18.970 & 19.218 & 18.279 & $\mathrm{~S} 15$ & $\mathrm{QSO}, z_{\mathrm{em}}=2.16$ \\
\hline \multicolumn{6}{|c|}{ Unclassified } \\
\hline $004504.34-724449.9$ & 17.906 & 18.245 & 17.356 & $\mathrm{~S} 22$ & F star \\
\hline 004702.90-730800.7 & 18.036 & 18.506 & 17.346 & $\mathrm{~S} 23$ & F star \\
\hline $005039.12-724154.3$ & 18.871 & 19.138 & 18.305 & S24 & F star \\
\hline 005137.19-731429.2 & 17.221 & 17.240 & 16.973 & S25 & Be star \\
\hline
\end{tabular}

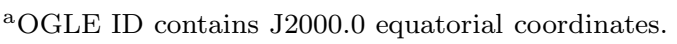

${ }^{\mathrm{b}}$ Magnitudes from Udalski et al. 1998.

${ }^{\mathrm{c}}$ Object identified in MACHO database by Geha et al. 2002.

${ }^{d}$ Near object 171 in Kahabka et al. 1999 and object 182 in Haberl et al. 2000.

e Near object 203 in Kahabka et al. 1999 and object 338 in Haberl et al. 2000. 
OGLE database. The method is very efficient. As expected, the contaminants in the list of candidates were predominantly early type stars in the SMC. Qualitatively, we do not see any obvious trends or differences between the light curves of quasars and stars. In Figure 2 we show the OGLE light curves of the QC objects, arranged similarly to Fig. 1.

It appears, however, that the source colors will help in improving the candidate lists. In Figure 3 we show the color-color diagram for the twelve sources presented in this paper, plus twenty four Eyer's QCs from the LMC region which were identified by Geha et al. (2002) and Huchra (2002, private communication). One can clearly see that variability-selected stars and quasars occupy different regions in the color-color space. We note here that the Faint Sky Variability Survey (Groot et al. 2002) data seem to show a similar effect.

In Fig. 3 we also show the known X-ray selected quasars in both the LMC and the SMC for which OGLE photometry is available. Those quasars also separate well from the stars. We note that one of Eyer's selection criteria for quasar candidates was a color cutoff, $V-I<0.9$, but this criterion was not applied to the X-ray-selected quasars before they were identified. They are typically redder than Eyer's objects.

As mentioned earlier, until very recently only a handful of confirmed quasars were known in the general direction of the SMC (Crampton et al. 1997; Tinney et al. 1997). All those quasars are located in fairly sparse stellar fields away from the center of the SMC, which limits their applicability to studies of SMC proper motion or investigations of absorption properties of the SMC. Quasars presented in the present paper and objects from Geha et al. (2002) lie behind the dense parts of the SMC. Note that those quasars - extremely interesting and useful objects in their own right - are in reality byproducts of monitoring surveys, unrelated to the original scientific goals of the surveys. It is an excellent example that such projects can lead to unexpected, yet very valuable results.

Geha et al. (2002) independently identified two of our quasars. We note that those two objects are the largest redshift quasars among our five, but this most likely is just a coincidence, not a result of the difference in the methods applied by Geha et al. and Eyer. Geha et al.'s quasar redshifts span a large redshift range. We note, however, that two methods, which, after all, are based on a similar concept, apparently have a rather small intersection in the final candidate lists: there are only ten objects in common in both the SMC and the LMC. This fact and the fact that X-ray-selected quasars are redder than the variability-selected objects indicate that both techniques are conservative and both will miss some quasars. The two methods should therefore be considered as complementing one another, rather than as competing.

We note here that the same two quasars lie relatively close to ROSAT X-ray sources 


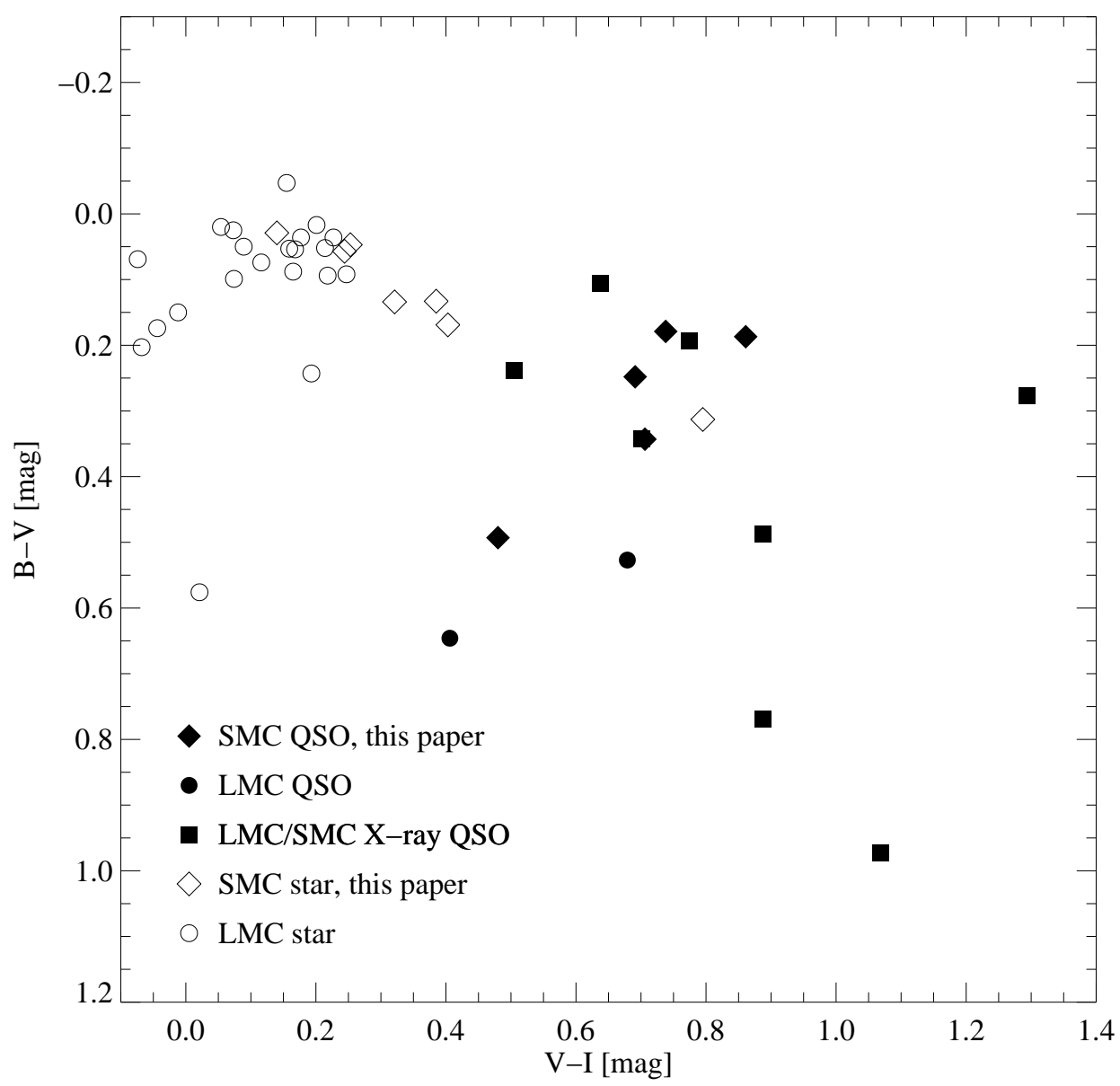

Fig. 3.- The $V-I$ versus $B-V$ color-color diagram for Eyer objects for which identifications are available. Filled and open diamonds show quasars and stars, respectively, presented in this paper. The circles show quasars (filled) and stars (open) that were included in Eyer's quasar candidate list for the LMC and were identified by Geha et al. (2002) and Huchra (2002, private communication). Eyer candidate lists contain objects for which $V-I<0.9$. We also show (solid squares) the quasars identified by us via matching OGLE sources with serendipitous X-ray sources from the Chandra X-ray Observatory (Dobrzycki et al. 2002; 2003, in preparation); Eyer's color criterion was not applied for those objects. 
listed in Kahabka et al. (1999) and Haberl et al. (2000), although neither one of the X-ray sources was classified as a probable QSO.

One of the quasars identified in this paper, QSO J003850.79-731053.1 $\left(z_{\mathrm{em}}=0.28, V=\right.$ 17.7) is a very promising candidate for studies of absorption in the SMC. Its brightness should enable good spectroscopy with the Cosmic Origin Spectrograph aboard the Hubble Space Telescope. The other four quasars and quasars from Geha et al. (2002) are very well positioned to be reference points for SMC proper motion studies. We add here that we also identified six other X-ray selected quasars behind the dense parts of the SMC; we will present them in the forthcoming paper (Dobrzycki et al. 2003, in preparation).

We were somewhat surprised to find a G-type star among Eyer's QC objects, especially since he did utilize color selection in constructing the list of candidates. However, it was also the faintest of the observed objects, for which the photometry is likely rather uncertain.

Excellent efficiency of the variability-based method bodes well for searches of quasars behind other dense stellar fields for which monitoring photometry databases are available. A first successful search has already been published by Geha et al. (2002), who found 38 QSOs behind the LMC in the MACHO database.

The paper by Eyer (2002) contains a list of 118 QSO candidates behind the LMC. At present, identifications are known for $\sim 25$ of them (Geha et al. 2002; Huchra 2002, private communication). As noted earlier, the color-color diagram for identified Eyer's QSO candidates indicates that quasars are typically redder in $V-I$ than QCs that turned out to be stars (Fig. 3). Remaining objects from Eyer's LMC candidate list split roughly evenly between the quasar and stellar regions in the color-color diagram, indicating that the spectroscopic followup should yield a large number of quasars.

Geha et al. (2002) noted that their quasars tend to be bluer when they brighten. OGLE time coverage in $B$ and $V$ filters is much sparser than in $I$, which, combined with relatively small size of our sample, does not allow us to make quantitative statements of that nature. We do note, however, that this effect is present in the brightest of our quasars, QSO J003850.79-731053.1: the well defined features in the light curve of this object (top left panel on Fig. 2) have corresponding changes in the $V-I$ color consistent with the effect seen in MACHO quasars.

Another extremely interesting region to search for quasars where the method could be applied is the Galactic Bulge. To our knowledge, no quasars have been identified so far in the vicinity of the Galactic center. There are, however, several regions where interstellar extinction is quite low $\left(A_{V}<3\right)$ and where one could, in principle, see quasars. The best known such area is Baade's Window (e.g. Stanek 1996), but there are several other. 
Identifying quasars behind the Galactic Bulge would be very valuable. Recently, Sumi, Eyer, \& Woźniak (2002) have shown that there is a statistically significant difference between proper motions of faint versus bright red clump stars in one of the OGLE bulge fields. Finding fixed reference points for this study, which quasars could provide, would be an extremely interesting result.

On one hand, the search toward the Galactic bulge will be made easier by the fact that there will be few early type stars, which in the Magellanic Clouds are the primary contaminants in the candidate lists. On the other hand, the quasars will be considerably reddened even in the low extinction windows, making them less conspicuous as blue objects. Also, the surface density of objects that need to be analyzed will be very large, and as a result there will likely be a sizeable number of artifacts, etc., which will contaminate the candidate lists.

We would like to thank L. Eyer, B. Paczyński and J. Stocke for helpful discussions, J. Huchra for observing the LMC candidates, and the referee, M. Geha, for helpful comments and, especially, for her suggestion to explore the information contained in the color-color diagram. AD acknowledges support from NASA Contract No. NAS8-39073 (CXC). LMM was supported by the Hubble Fellowship grant HF-01153.01-A from the Space Telescope Science Institute, which is operated by the Association of Universities for Research in Astronomy, Inc., under NASA contract NAS5-26555. 


\section{REFERENCES}

Alard, C., \& Lupton, R. H. 1998, ApJ, 503, 325

Anguita, C., Loyola, P., \& Pedreros, M. H. 2000, AJ, 120, 845

Blanco, V. M., \& Heathcote, S. 1986, PASP, 98, 635

Crampton, D., Gussie, G., Cowley, A. P., \& Schmidtke, P. C. 1997, AJ, 114, 2353

Dobrzycki, A., Groot, P. J., Macri, L. M., \& Stanek, K. Z. 2002, ApJ, 569, L15

Eyer, L. 2002, AcA, 52, 241

Geha, M., et al. 2002, AJ, in press (astro-ph/0209513)

Groot, P. J., et al. 2002, MNRAS, in press (astro-ph/0210416)

Haberl, F., Filipović, M. D., Pietsch, W., \& Kahabka, P. 2000, A\&AS, 142, 41

Hamuy, M., Walker, A. R., Suntzeff, N. B., Gigoux, P., Heathcote, S. R., \& Phillips, M. M. 1992, PASP, 104, 533

Hawkins, M. R. S. 1983, MNRAS, 202, 571

Kahabka, P., de Boer, K. S., \& Brüns, C. 2001, A\&A, 371, 816

Kahabka, P., Pietsch, W., Filipović, M. D., \& Haberl, F. 1999, A\&AS, 136, 81

Meusinger, H. \& Brunzendorf, J. 2001, A\&A, 374, 878

Rengstorf, A. W., et al. 2001, AAS Meeting, 199, 138.07

Stanek, K. Z. 1996, ApJ, 460, L37

Sumi, T., Eyer, L., \& Woźniak, P. R. 2002, MNRAS, submitted (astro-ph/0210381)

Tinney, C. G., Da Costa, G. S., \& Zinnecker, H. 1997, MNRAS, 285, 111

Udalski, A., Kubiak, M., \& Szymański, M. 1997, AcA, 47, 319

Udalski, A., Szymański, M., Kubiak, M., Pietrzyński, G., Woźniak, P., \& Żebruń, K. 1998, AcA, 48, 147

Woźniak, P. R. 2000, AcA, 50, 421

Żebruń, K., et al. 2001, AcA, 51, 317 